\title{
Pratiques géotouristiques et interprétation de la nature dans les géoparcs chinois : entre tensions et hybridation des cultures
}

\section{Yi Du et Yves Girault}

\section{(2) OpenEdition}

Édition électronique

URL : https://journals.openedition.org/ere/3393

DOI : $10.4000 /$ ere.3393

ISSN : 2561-2271

\section{Éditeur}

Centr'ERE

\section{Référence électronique}

Yi Du et Yves Girault, «Pratiques géotouristiques et interprétation de la nature dans les géoparcs chinois : entre tensions et hybridation des cultures », Éducation relative à l'environnement [En ligne], Volume 15 - 1 | 2019, mis en ligne le 23 septembre 2019, consulté le 18 octobre 2021. URL : http:// journals.openedition.org/ere/3393 ; DOI : https://doi.org/10.4000/ere.3393

Ce document a été généré automatiquement le 18 octobre 2021

\section{(c) (†) (8)}

La revue Éducation relative à l'environnement est mise à disposition selon les termes de la Licence Creative Commons Attribution - Pas d'Utilisation Commerciale 4.0 International. 


\title{
Pratiques géotouristiques et interprétation de la nature dans les géoparcs chinois : entre tensions et hybridation des cultures ${ }^{1}$
}

\author{
Yi Du et Yves Girault
}

Depuis les années 1980, des travaux effectués dans des cercles universitaires, tant en Europe, en Amérique du Nord, qu'au Japon, ou à Taiwan ont montré que la perception «d'un pays immuable, imperméable à l'écoulement du temps" qu'avaient des historiens du $19^{\text {ème }}$ siècle sur la Chine ne pouvaient plus être retenue (Elliott, 2006, p. 1447). Ainsi comme le remarque l'historien de l'environnement Mark Elvin, «il n'y a pas de point de vue unique sur la nature que l'on puisse qualifier de "point de vue chinois ". Il n' y a même pas de spectre, plutôt un kaléidoscope de fragments de points de vues dont la plupart reflètent quelque chose des autres fragments" [traduction libre] (Elvin, 2004, p. 413). En se basant sur une sélection de textes issus d'une large bibliographie existante, nous avons centré notre analyse ${ }^{2}$ sur l'évolution des rapports à l'environnement induite d'une part par des pratiques culturelles spécifiques à l'Asie (dont le confucianisme et le taoïsme) et d'autre part, par l'intégration d'éléments de la culture occidentale. Trois périodes historiques sont généralement retenues. Durant la première, celle de la Chine dynastique avant le $19^{\text {ème }}$ siècle, ont émergé les mouvements de paysage, le développement du tourisme lettré $e^{3}$ la pratique d'inscriptions dans les paysages $^{4}$ et le culte de la montagne ${ }^{5}$. Vers la fin du $19^{\text {ème }}$ et au début du $20^{\text {ème }}$ siècle (selon Lin, 2009, p. 20), l'évolution sémantique du terme «nature» illustre le long processus d'intégration d'un système épistémique occidental en Chine. C'est au cours de la dernière période, celle de la Chine contemporaine, qu'a été mis en place le modèle de « réserve naturelle » qui doit ses origines à la recherche scientifique dans les années 1950. Avec le déclin du Lyssenkisme ${ }^{6}$, les réserves naturelles, qui étaient perçues comme un «laboratoire de la nature » (Songster, 2004 p. 73), ont progressivement été transformées en «laboratoire dans la nature " par les biologistes qui privilégiaient l'observation de la nature vierge (op.cit., p. 75). 
2 Xu et coll. précisent " qu'en construisant une nouvelle Chine, la culture traditionnelle n'a pas seulement été ignorée mais activement réprimée alors que Mao cherchait à détruire les "Quatre Vieux ${ }^{7}$. Spécifique aux principes de la protection de l'environnement qui opposent le plus souvent l'Homme à son environnement, le système occidental de zones protégées qui a été imposé, compte tenu du pouvoir de l'État, a modifié tout le modèle de protection de l'environnement (Xu, Cui, Sofield etLi, 2014, p. 1133). À partir de la fin des années 1970, sous la double influence des nouvelles tendances de décentralisation et de la montée de l'industrie touristique, le système national de réserves naturelles a évolué pour donner naissance aux «zones paysagères » et aux "parcs forestiers ». À la même période, la Chine a intégré les programmes internationaux de patrimonialisation de la Nature avec la création des premières réserves de biosphères puis des sites du patrimoine mondial. À l'instar des occidentaux, les responsables de ces aires protégées se sont donnés progressivement comme objectif de développer le tourisme local (à l'exception des premières réserves naturelles) en adoptant un nouveau concept, l'écotourisme, qui a été introduit dans la littérature anglophone par Romeril (1985). En effet, depuis le début des années 2000 on constate que de nombreuses structures d'accueil des publics au sein d'aires protégées (réserves de biosphère, parcs nationaux) ou de géoparcs font la promotion du tourisme de nature qui nécessite notamment la création de structures d'accueil (musées, centres d'interprétation, sentiers d'interprétation, géoroutes, etc.). En partant du principe que nous pouvons faire un lien entre la fréquentation des aires protégées et le développement de l'écotourisme, la présentation de quelques taux de fréquentation est riche d'enseignement sur le développement de cette activité notamment en Asie du sud Est. Ainsi, au sein des Parcs nationaux des États-Unis on dénombrait en 2018, 318,2 millions $^{8}$, et en 2016 ce sont 890 millions de visiteurs qui ont fréquenté les aires paysagères nationales chinoises ( $\mathrm{Wu}$ et $\mathrm{Cai}, 2006)$ et 45 millions de visites ont été effectuées dans les Parcs nationaux coréens en 2015 (Choe, Schuett, \& Sim, 2017). En Chine, cette ouverture du marché de l'écotourisme a apporté de nouvelles opportunités aux territoires labélisés Unesco Global Geopark (UGG) car les touristes chinois, très influencés par leur culture traditionnelle, privilégient le tourisme de montagne et l'on dénombre ainsi en 2018 une moyenne annuelle de 12 millions de visiteurs par géoparc. Dans le cadre de ce numéro thématique sur l'éducation à l'environnement au sein des aires protégées et des musées, il nous a semblé intéressant d'analyser les intérêts des acteurs de la construction des géoparcs en Chine au regard notamment des prescriptions de l'UNESCO. Après avoir présenté les principaux courants de l'écotourisme et du géotourisme et le contexte d'évolution des géoparcs en Chine, nous analysons donc les pratiques hybrides de l'interprétation du patrimoine naturel au sein des géoparcs chinois.

\section{De l'écotourisme au géotourisme}

\section{Émergence et évolution de l'écotourisme}

3 Selon Blamey (2001) l'écotourisme présente trois caractéristiques essentielles.

-L'écotourisme se focalise sur les écosystèmes et la mégafaune endémique non captive ou charismatique indigène (baleine, panda géant, éléphant, lion etc.) et parfois sur la mégaflore 
charismatique (dont les séquoias et les baobabs) et des mégalithes (dont les volcans et les falaises).

- Les motivations écotouristiques sont largement fondées sur l'apprentissage (éducation formelle et non formelle) et une sensibilisation à l'appréciation personnelle esthétique ou spirituelle. L'interprétation in situ est assurée au moyen de diverses formes de médiation (centres d'accueil, guides touristiques, panneaux de signalisation permanents et des guides d'interprétation).

- L'écotourisme se distingue enfin par ses objectifs de durabilité ce qui implique le respect de protocoles de planification et de gestion qui visent à minimiser les coûts environnementaux, socioculturels et économiques de ces pratiques tout en maximisant les avantages associés en terme d'éducation non formelle et de conservation des écosystèmes.

4 Cette définition est partagée par de nombreux auteurs dont Donohoe et Needham, (2006) et Weaver et Lawton (2007) qui précisent que, dans le monde occidental, il y a généralement trois critères communs pour définir l'écotourisme: les produits et attractions écotouristiques qui sont axés sur la conservation, la nécessité de l'interprétation et, enfin, la prise en compte de la durabilité des installations sur les plans écologique, économique et socioculturel. L'écotourisme tel qu'il a été introduit en Chine en 1992 définissait, selon Sofield et Li (2007), tout développement et activité touristique située dans un cadre naturel. Buckley et coll. (2008), qui ont comparé le concept chinois de «shengtai lüyou » au concept occidental d'écotourisme ont, pour leur part, identifié trois différences : a) l'écotourisme en Chine a un rôle de promotion de la santé humaine (pratiques de plein air) ; b) il a une prédilection pour la valorisation du patrimoine naturel par l'art (roches gravées et création de pavillons) ; c) il ne comporte aucune limitation d'échelle géographique.

5 Ainsi, pour de nombreux touristes chinois, vivre l'expérience de la nature ne doit nullement inclure des activités nécessitant un effort physique ou sportif. À l'opposé, ils veulent réaliser une expérience de vie idéale représentée en peinture "shan shui » $\mathrm{Xu}$ et coll., 2013, cité dans Xu et coll., 2014, p. 1140). Ils veulent donc disposer, dans les zones naturelles, d'un accès facile, de confort, plutôt que de pratiquer un exercice physique. Ils préfèrent écouter des histoires divertissantes s'inspirant de nombreuses sources (mythes et légendes locales, extraits pertinents de la vaste collection de poèmes et de classiques de la Chine, et contes de l'histoire) plutôt que de découvrir et/ ou acquérir des connaissances scientifiques comme cela est proposé dans de nombreux sites naturels occidentaux qui présentent aux publics des informations sur la biologie, la géologie, le comportement animal, etc.) (Xu et coll., 2013, cité dans Xu et coll., 2014, p. 1140). En conséquence, à l'opposé des aménagements relativement rares et discrets réalisés en Occident au sein des sites naturels, ce sont des routes, des chemins de béton, des chemins de fer, des escaliers aux milliers de marches menant aux sommets des montagnes, des hôtels et restaurants qui sont construits pour faciliter l'accès, la satisfaction et le confort du tourisme de montagne. Ces divers aménagements permettent au plus grand nombre d'atteindre des sommets, ce qui est perçu comme l'une des activités jugées les plus attrayantes, car issue d'une tradition culturelle remontant à plusieurs milliers d'années. Par exemple, le célèbre poème chinois «When shall I reach the top and hold all mountains in a single glance » de $\mathrm{Du} \mathrm{Fu}$, incite les touristes à grimper jusqu'au sommet de la montagne pour admirer la vue panoramique. Cela est particulièrement vrai des cinq montagnes les plus vénérées: le Tai-shan oriental, le Heng-shan méridional (montagne équilibrée/harmonie), le Hua-shan occidental, le 
Heng-shan septentrional (montagne éternelle) et le Song-shan central (Xu et coll., 2014, p. 1142). On peut noter une autre illustration de cette harmonie avec la nature par le fait que ces montagnes sont parsemées de rochers sur lesquels sont gravées les calligraphies de poèmes célèbres, anciens ou nouveaux. Dans de nombreuses réserves naturelles, les visiteurs peuvent également trouver des arbres de souhaits décorés (Weller, 2006), dont les plus vieux, en particulier les espèces de ficus, sont censés détenir des pouvoirs mystiques et, dans la croyance bouddhiste, ils peuvent même parfois être sacrés.

Ce développement de l'écotourisme, en occident et en Chine, mobilise le courant de l'interprétation pour des objectifs très différents. Ainsi, selon Xu, Cui, Ballantyne et Packer (2013) si depuis les années 1970 dans la société occidentale, l'interprétation a joué un rôle important dans l'amélioration de l'appréciation du milieu naturel, le développement d'attitudes et de comportements respectueux de l'environnement, il en est tout autrement en Chine car ce rapport à la nature est déjà largement prégnant dans la culture traditionnelle. À l'opposé, l'interprétation de la nature promue par les instances gouvernementales privilégie bien plus, comme nous allons le préciser, la présentation d'une information scientifique pour contribuer à combattre les superstitions et les pseudo-sciences (Shi et Zhang, 2012, p. 91). Cependant, les recherches que ces auteurs ont menées dans la Réserve naturelle nationale Danxiashan et au sein du Géoparc montrent que si les outils d'interprétation se basant sur l'approche scientifique "occidentale" avec des panneaux d'information, un centre d'information et un musée géologique sont très peu usités et donc inefficaces, d'autres documents d'information comprenant des descriptions figuratives ou métaphoriques du paysage et de nombreux qualificatifs qui tendent à une exagération de la beauté du paysage semblent privilégiés par les touristes locaux (Xu et coll., 2013, p. 125).

7 Nous allons donc analyser, dans les lignes qui suivent, de quelles façons ces pratiques très populaires de «tourisme de montagne » en Chine peuvent être prises en compte dans les approches contemporaines du géotourisme et des courants d'interprétation de la nature.

\section{Émergence et évolution du géotourisme}

8 Selon Dowling et Newsome (2006, p. 6), le géotourisme peut être considéré comme faisant partie du tourisme de nature et de l'écotourisme. Il s'agit néanmoins d'une forme spécialisée de tourisme dans la mesure où le géosite, soit une formation géologique exceptionnelle localisée qui illustre et révèle certains aspects de l'histoire de la Terre ou de la vie et qui fait d'elle une référence et donc un élément du patrimoine géologique, est au centre de l'attention. Ainsi, les quatre premiers géoparcs européens créés à l'aide du programme de financement européen Leader en 1999 ont utilisé ce concept de géotourisme comme support du développement local. C'est la raison pour laquelle, dans un article récent, nous avons voulu montrer qu'avec la création du Réseau européen des Géoparcs (EGG) puis du Réseau mondial des Géoparcs de l'UNESCO (UNESCO Global Geopark que nous noterons par la suite UGG), les définitions retenues pour qualifier le géotourisme ont évolué (cf. tableau 1). 
Tableau 1 : Typologie des principales approches du géotourisme d'après Gonzalez, Du, Read et Girault (2017, p .12)

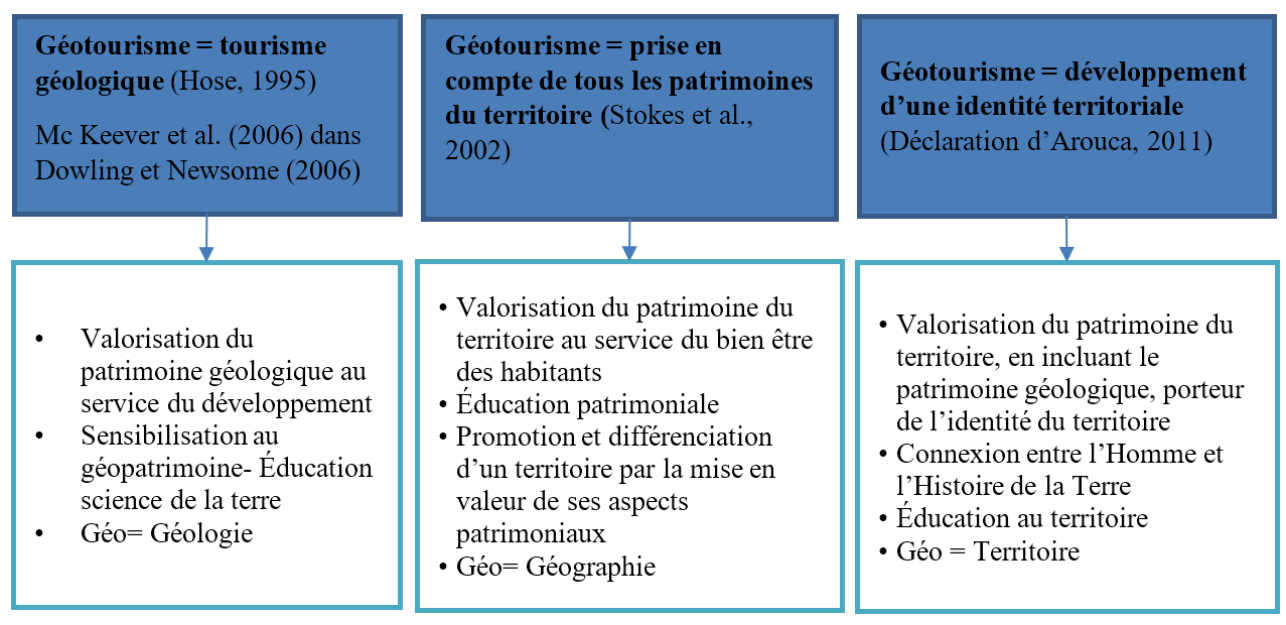

9 Cette typologie des diverses approches du géotourisme laisse entrevoir trois courants d'enseignement / d'éducation. Le premier basé sur le « deficit model » qui implique de proposer aux visiteurs/touristes une information scientifique conséquente, le deuxième qui se rapporte plus à l'éducation au patrimoine (sensibilisation/ valorisation) et le dernier qui se rapporte à l'un des courants de l'éducation aux territoires basé plus explicitement sur l'interprétation (Gonzalez et coll., 2017).

10 Avant d'analyser dans quelle mesure le géotourisme en Chine s'intègre dans la typologie présentée ci-dessus, nous allons présenter succinctement le contexte d'émergence des géoparcs dans le monde pour nous focaliser par la suite sur la Chine.

\section{Géoparc mondial en Chine : histoire, valeurs et objectifs}

11 Suite à la Déclaration des droits de la mémoire de la Terre (1991), c'est en 1999 qu'a été proposée une nouvelle initiative pour promouvoir un réseau mondial de géoparcs9. Un géoparc était alors défini comme "une zone spéciale renfermant des éléments d'une rareté, d'une beauté ou d'un intérêt géologique particulier. Ces éléments devront être représentatifs de l'histoire géologique d'une région donnée ainsi que des évènements et processus qui l'ont formée» (UNESCO 156 EX/11Rev, p. 2). Cette initiative précise également qu'en plus d'offrir « des possibilités de recherche scientifique et d'éducation écologique au sens large, un géoparc peut être un puissant facteur de développement local durable. » Considérant le concept de géoparc comme une construction dynamique continue, nous avons montré que ce processus peut se décomposer en trois étapes ( Du et Girault 2018). Dans un premier temps (1996-2004), plusieurs scénarios d'intégration des géoparcs dans les programmes existants - Patrimoine mondial, L'Homme et la biosphère (MAB) ou Programme international de corrélation géologique (PICG) - ont été examinés par l'UNESCO, puis progressivement rejetés. La deuxième phase (2004-2010) a vu le développement indépendant et la mise en réseau des géoparcs en Europe et en Asie. Au cours de la troisième phase (2011-2015), l'UNESCO a finalement relancé les négociations pour officialiser le programme international des géosciences et des géoparcs (PIGG) afin d'offrir plus de soutien à une répartition plus équitable des géoparcs sur toute la planète. 
12 En Chine, jusqu'en 1999 aucune des 86 réserves de géosites ${ }^{10}$ n'avait été créée à l'image d'un géoparc. Chen Anze et coll. (2015), précisent que la proposition de création de géoparcs n'a été acceptée par l'État qu'après le lancement de l'initiative de l'UNESCO en 1999. De fait, c'est en 2000 qu'a été désigné le Groupe d'experts sur les géosites nationaux qui allait assister le ministère du Territoire et des Ressources (MTR) dans la sélection des 11 premiers géoparcs nationaux Chinois (Du, Girault 2018). Ce groupe a également publié «le Plan national sur la protection du patrimoine géologique (2001-2010) », dont l'objectif était d'établir, au cours des dix années qui suivirent, 310 réserves géosites (géoparcs) dont entre 5 à 8 classées dans la liste du géopatrimoine mondial ${ }^{11}$.

Depuis, la construction des géoparcs s'est largement développée en Chine pour atteindre, en 2013, le nombre de 420 dont 184 géoparcs nationaux d'une surface totale de 11989960 hectares et 31 géoparcs mondiaux classés dans le GGN. En mai 2018, la Chine est le pays qui possède le plus grand nombre de géoparcs mondiaux soit 37 sur 140 UGG.

14 Les premiers documents normatifs sur les géoparcs nationaux étant inaccessibles, nous avons néanmoins trouvé une définition officielle d'un géoparc dans « la Notification sur la nomination des géoparcs nationaux", document publié par le ministère du Territoire et des Ressources en 2000 :

Un géoparc est un parc scientifique possédant des valeurs scientifiques spéciales (attributs naturels rares, superbes valeurs esthétiques) et un géopaysage thématique d'une taille et d'une aire de répartition appropriées - avec des valeurs écologiques, historiques et culturelles intégrant ses paysages naturels et culturels dans le but de conserver les géosites et soutenir le développement durable dans le respect de l'économie locale, de la culture et de l'environnement - et de fournir un service public en faisant une promotion de la science par le biais de visites guidées, de loisirs de vacances, de la promotion de la santé et du rétablissement de la promotion et de l'éducation scientifique et d'autres activités de loisirs culturels. Un géoparc se situant sur une importante zone protégée et bénéficiant de ce fait d'un environnement écologiquement sain est une base pour la recherche et la promotion des géosciences ${ }^{12}$. [Traduction libre de la version anglaise de Chen et coll. (2015, p. 225)]

Les auteurs chinois qui publient en anglais ont eu davantage tendance à décrire les missions des géoparcs en Chine par les fonctions similaires de celles des géoparcs européens, à savoir la conservation des géosites, la promotion des géosciences, et le développement durable (Chen, $\mathrm{Lu}$ et $\mathrm{Ng}, 2015, \mathrm{p}$. 226). Certes, si ces trois aspects sont tous présentés dans cette définition, les objectifs et l'interprétation des principes ne nous semblent cependant pas identiques. Par rapport aux géoparcs européens, la définition des géoparcs nationaux chinois semble bien moins focalisée sur la prise en compte des communautés locales ou l'identité du territoire pour se centrer davantage sur les valeurs esthétiques et le paysage. Hou et coll. (2004, p. 50) jugent ainsi que la valeur esthétique appliquée au tourisme est l'une des deux valeurs essentielles du géoparc, l'autre étant la valeur scientifique. La prise en compte de ces deux valeurs, l'une ancrée dans la culture chinoise (l'aspect esthétique des paysages), l'autre dans la modernité (l'aspect scientifique) va immanquablement induire quelques tensions en termes notamment d'interprétation du paysage. 


\section{Les pratiques de l'interprétation du patrimoine au sein des géoparcs en Chine}

16 Comme nous l'avons déjà précisé, Xu et coll. (2014) ont montré que l'interprétation du patrimoine culturel, qui est en Chine un phénomène récent, privilégie une approche cognitive axée sur la présentation d'informations scientifiques. Cette utilisation du terme "interprétation », qui ne correspond nullement au sens premier décrit par les précurseurs de ce courant comme Enos Miles ou Freemand Tilden s'apparente bien plus, selon Shi et Zhang (2012) et Wu (2016), au courant de la « science popularization » ( kepu 科普 en chinois) qui est entré dans la constitution chinoise en 1982 et qui s'est par la suite progressivement développé pour atteindre son apogée en 2002 avec la promulgation de la loi sur la popularisation des sciences et des technologies. Shi et Zhang (2012) précise à cet égard que la "science popularization» a servi à la construction de deux "civilisations»: le développement économique qui va induire l'émergence d'une "civilisation matérielle» et, pour des raisons idéologiques, le développement social d'une « civilisation spirituelle socialiste $»^{13}$.

17 Ainsi dès 2006, dans le Manuel de la construction des géoparcs nationaux (2006), le ministère du Territoire et des Ressources précisait que le deuxième rôle du géoparc (derrière la protection du patrimoine géologique) était sa contribution à «la construction de la civilisation spirituelle de la société ».

La création de géoparcs est une mesure importante pour défendre la science et se débarrasser de la superstition. La construction de géoparcs a pour objectif principal de populariser les connaissances géoscientifiques, propager la vision matérialiste $\mathrm{du}$ monde et s'opposer à la superstition féodale. Il faut y développer non seulement l'interprétation culturelle du paysage naturel, mais aussi l'interprétation géoscientifique, ainsi le géoparc est à la fois divertissant et scientifique. [Traduction libre du texte chinois]

18 L'instauration de ce courant de la "science popularization " peut-elle répondre aux attentes de la majorité des touristes chinois qui visitent des géoparcs? Nous voulons donc prolonger la réflexion engagée par Xu et coll. (2014) en analysant, pour notre part, plus spécifiquement les problèmes engendrés par la pratique de l'interprétation dans les UGG en Chine. Cette pratique semble confrontée à un problème majeur, souvent perçu comme une cohabitation entre le modèle imposé par les politiques promues par l'Occident (principalement les programmes UNESCO) et des intellectuels chinois qui privilégient la «science popularization » d'une part et, d'autre part, le fait que la culture traditionnelle exerce encore une influence significative sur les rapports que les Chinois « ordinaires» entretiennent avec la Nature (Xu et coll., 2014, p. 1142). En nous focalisant sur la troisième perspective de Pieterse (2009, p. 44) qui stipule que «l'hybridation, signifie que ce qui se passe est un processus de mélange ou d'hybridation culturelle entre les lieux et les identités ", nous souhaitons analyser ce qui semble s'opérer au sein des géoparcs mondiaux et nationaux chinois.

19 Pour contribuer à répondre à notre questionnement sur l'hybridation des cultures, nous avons donc analysé l'influence de l'UNESCO en mobilisant les principales recommandations données par les évaluateurs de UGG aux 17 géoparcs chinois réévalués en 2016 et 2017. Étant donné l'importance fondamentale pour tous les responsables Chinois de géoparc d'obtenir une évaluation favorable, nous allons également montrer que tous les travaux d'aménagement, et de réaménagement, sont 
effectués en fonction des critères d'évaluation fixés par l'UNESCO et sont réalisés par vagues quadriennales juste avant chaque évaluation.

\section{Mode d'évaluation pour les géoparcs mondiaux UNESCO (UGG)}

Pour effectuer notre analyse nous mobilisons la procédure d'évaluation retenue par High et Nemes (2007) pour leurs travaux réalisés sur le programme de développement rural européen LEADER. Celle-ci souligne les différentes logiques d'évaluation suivant les différentes logiques entre le développement exogène et le développement endogène.

Tableau 2 : Comparaison entre l'évolution exogène et endogène d'après High et Nemes (2007)

\begin{tabular}{|c|c|c|}
\hline & Évaluation exogène & Évaluation endogène \\
\hline $\begin{array}{l}\text { Logique } \\
\text { d'évaluation }\end{array}$ & $\begin{array}{l}\text { - Top-down et standardisée } \\
\text { - Mesure basée sur les méthodes } \\
\text { expérimentales des sciences } \\
\text { naturelles } \\
\text { - Focus sur les mesures } \\
\text { financières et autres mesures } \\
\text { quantitatives. } \\
\end{array}$ & $\begin{array}{l}\text { - Intégrée et intersubjective } \\
\text { - Compatible avec les modes de } \\
\text { connaissance du système } \\
\text { heuristique local } \\
\text { - Basée sur les interactions } \\
\text { continues des êtres sociaux dans } \\
\text { un contexte particulier. }\end{array}$ \\
\hline Communication & $\begin{array}{l}\text { - L'information est perçue comme } \\
\text { des données transférables en } \\
\text { application du modèle de } \\
\text { Shannon (codage, signalisation } \\
\text { et transfert) }\end{array}$ & $\begin{array}{l}\text { - Communication coopérative et } \\
\text { communautaire basée sur la } \\
\text { métaphore de « dance-rituel » de } \\
\text { la communication (Krippendorff, } \\
\text { 1993, p.13-15). }\end{array}$ \\
\hline Épistémologie & $\begin{array}{l}\text { - Épistémologie réaliste, } \\
\text { concevant l'information comme } \\
\text { un bien }\end{array}$ & $\begin{array}{l}\text { - Épistémologie constructiviste } \\
\text { exprimée dans des approches telles } \\
\text { que « l'apprentissage localisé » et } \\
\text { « l'apprentissage social » }\end{array}$ \\
\hline
\end{tabular}

Bien que les quatre premiers géoparcs aient pu bénéficier du programme Leader (Du et Girault, 2018), nous pouvons déjà préciser que les UGG font l'objet d'une évaluation exogène top-down basée sur deux grilles d'évaluation standardisées. La première, appelée « self Evaluation Form » est composée de sept catégories de critères à évaluer, chacune disposant de 1000 points disponibles («points Available ») ${ }^{14}$ et ces points sont pondérés par le poids accordé ("weighting») à chaque partie pour obtenir la note finale de l'évaluation. La deuxième grille («progress evaluation form») porte sur les avancées que les responsables du géoparc ont pu effectuer pour améliorer leur candidature. Cette grille est composée de six sections, chacune d'elle donnant lieu à une note maximum sur un ensemble de 1000 points.

Le tableau 3 montre nettement une réorientation des focus d'évaluation entre la grille self evaluation form qui privilégie les deux axes géoconservation et gestion (les deux représentent $45 \%$ de la note finale) alors que le progress evaluation form (tableau 4) se focalise davantage sur la contribution au GGN et les activités de marketing et de promotion (52\% de la note globale). 
Tableau 3 : Pourcentage des points par catégorie selon la grille d'évaluation « Evaluation document A - self evaluation $»^{15}$

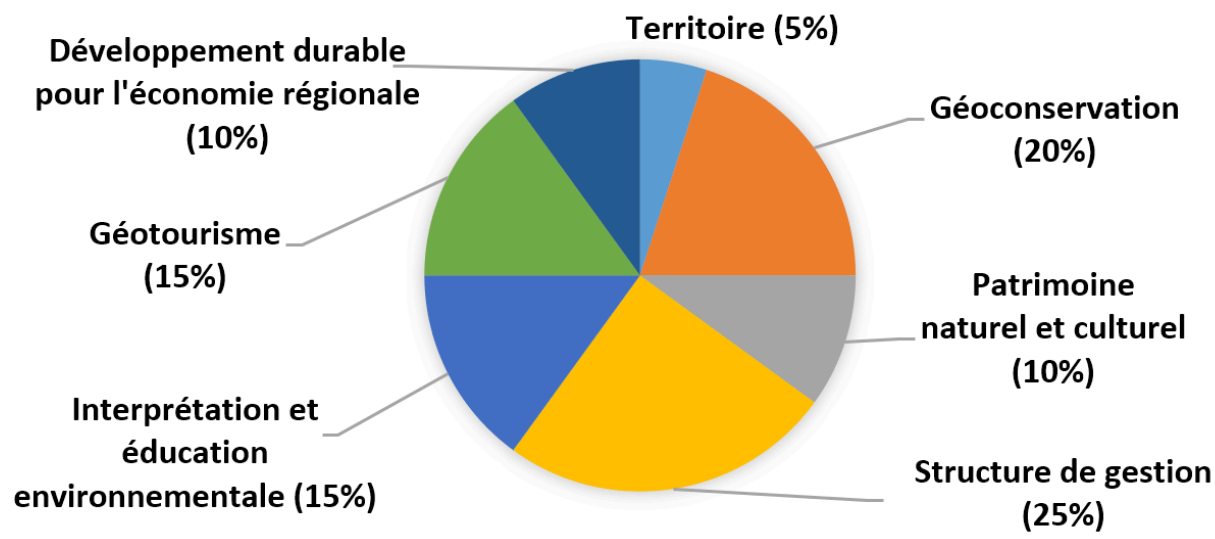

Tableau 4 : Pourcentage des points par catégorie selon la grille d'évaluation « Evaluation document $B$ - Progress evaluation form ${ }^{16}$

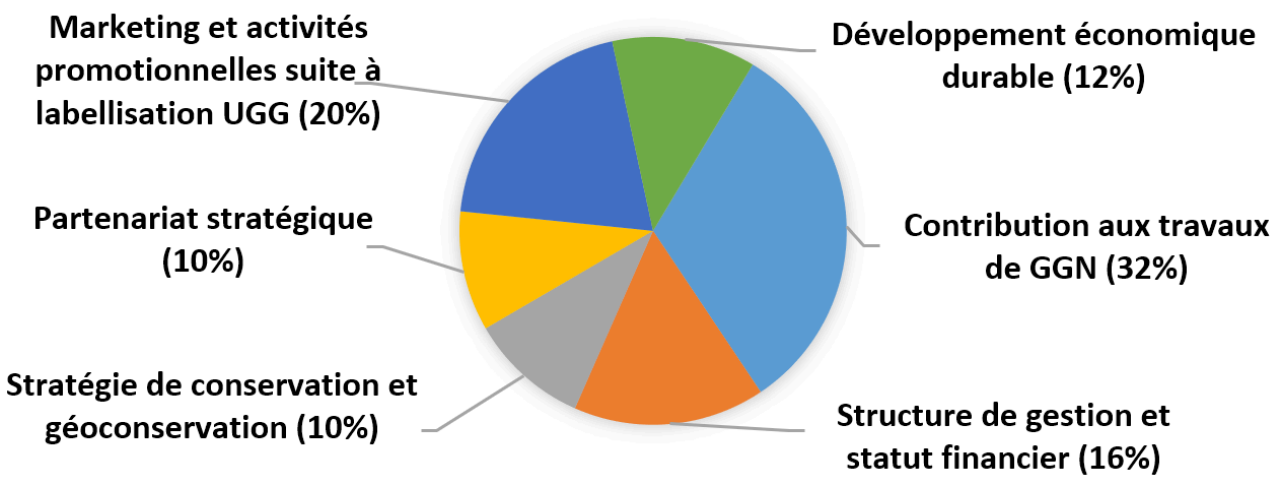

Depuis la ratification du programme $\mathrm{PIGG}^{17}$ en novembre 2015, un total de 17 UGG ont été évalués en Chine en 2016 et 2017. Dans les 17 lettres officielles signées par Patrick Mc Keever, alors secrétaire du PIGG, et envoyées aux responsables de géoparcs pour les informer de la décision de la revalidation (i.e. carte jaunes ou verte) nous avons collecté au total 144 recommandations. Parmi celles-ci nous avons retenu les 60 recommandations qui étaient relatives à l'interprétation et/ou à l'éducation pour lesquelles nous avons effectué une analyse thématique en la croisant avec des analyses de propos d'experts de géoparcs en Chine (propos issus de la revue de la bibliographie ou extraits d'entretiens ${ }^{18}$ ).

\section{Tensions et hybridation des cultures sur les finalités de l'interprétation promue au sein des géoparcs chinois}

Il est bon de rappeler qu'en 2013 « la carte jaune ${ }^{19}$ » obtenue par trois géoparcs chinois dans le cadre de leur évaluation a été largement médiatisée en tant qu'avertissement de la part de l'UNESCO pour souligner un déficit de « communication des géosciences aux publics » au sein de ces territoires.

Néanmoins il nous semble réducteur d'analyser cette situation comme une conséquence de tensions issues de normes euro-centriques car, comme nous l'avons 
déjà précisé, la nécessité de prendre en compte des activités de "science popularization » est également très largement privilégiée au niveau national et elle est donc revendiquée par des experts de géoparcs en Chine. Yin Hongfu, géologue et académicien, commente ainsi la décision de cette attribution des trois cartes jaunes à des géoparcs chinois :

Je suis complètement favorable à cette décision de l'UNESCO [...] Certains géoparcs aujourd'hui ont fait un travail déplorable. En termes de gestion, ils ne s'attachent qu'aux revenus économiques, et non à la communication scientifique ; en termes de promotion et d'interprétation, il n'y a que le paysage qui compte au détriment de la géologie, ce qui va à l'encontre de l'objectif fondamental de l'établissement d'un géoparc. [Traduction libre de l'extrait de l'article de presse en chinois ${ }^{20}$ ]

En soulignant très clairement ici son attachement au courant du tourisme géologique qui selon Dowling et Newson (2006), Mc Keever et coll. (2006) et Prosser et coll. (2011) privilégie le transfert de connaissances scientifiques, Yin Hongfu partage le point de vue d'experts de géoparcs en Chine pour qui le géoparc est avant tout un "science park $»^{21}$ qui doit lutter contre les superstitions.

Cette pratique de l'interprétation qui privilégie la «science popularization » est cependant peu adaptée à un large public parfois analphabète et le plus souvent dénué de formation scientifique ( $\mathrm{Du}$ et Girault, 2019). Ce point de vue est partagé par un géologue, qui participe à des travaux d'expertise dans des géoparcs chinois.

Si on veut sérieusement faire de la "science popularization " il faut accepter un écart entre les résultats de la recherche scientifique et le langage choisi qui ne doit pas être si rigoureux car l'objectif n'est pas le même. Pourtant cela n'est pas accepté car, pour le gouvernement, il faut absolument être exact et rigoureux. (Traduction libre d'un extrait d'entretien).

Cette critique des activités d'interprétation, telle qu'elles sont réalisées au sein d'UGG en Chine, est largement reprise par d'autres évaluateurs :

G2R9 G4R8, G5R7. L'utilisation d'un langage technique pour toutes les interprétations devrait être réduite et du matériel facile à comprendre devrait être développé pour le grand public et (G7R2, G8R4) rajoutent: y compris l'utilisation d'illustrations et d'autres images, et du matériel spécialement développé pour les enfants.

G1R1. Toute l'information géologique devrait être facile à lire et à comprendre par le grand public, dans tous les endroits et devrait être présentée d'une manière interactive et engageante. Dans la mesure du possible l'utilisation d'un langage spécialisé devrait être évitée en tout temps.

G9R2. Poursuivre le travail de simplification de la communication géologique vers le grand public, comme le démontre le nouveau centre d'accueil des visiteurs.

G15R2. Certains panneaux n'ont que du texte. Davantage de photos ou d'illustrations devraient être utilisées pour aider les visiteurs à comprendre la valeur géologique du site et à apprécier les merveilles géologiques.

Ainsi, la mise en place de cette approche de l'interprétation scientifique n'est pas aisée et les responsables des géoparcs sont souvent critiqués par les uns pour un manque de présentations géoscientifiques ${ }^{22}$ et, à l'opposé pour d'autres, pour l'utilisation d'un langage trop scientifique. La cause la plus souvent évoquée pour expliquer cette situation est, pour les premiers une offre touristique qui ne colle pas avec la «science popularization » et pour les autres elle réside dans l'absence de guide qualifié (Li et Yang, 2012 ; Geng et Wang, 2013). 

et pluridisciplinaire du territoire basée au besoin sur une approche sensitive et émotionnelle, n'est dû qu'aux pressions du gouvernement central et des scientifiques chinois? Il semble que ce soit plus complexe car, au niveau local, l'objectif des travaux d'interprétation est souvent éloigné des prérogatives de l'État centralisé. En effet, bien que l'objectif affiché soit de développer les activités de la "scientific literacy» du public, l'objectif réel de la mise en place de ces travaux d'interprétation est la quête d'une bonne évaluation réalisée soit au niveau national, soit par l'UNESCO. Ceci nous a été précisé par un autre spécialiste de géoparc chinois :

Les géoparcs mondiaux font beaucoup de travail en éducation en ce moment... pourquoi ? Parce que l'UNESCO leur demande. Souvent ils font ces travaux afin de répondre à l'évaluation. Comme les évaluateurs exigent beaucoup sur la visibilité [du label], ils travaillent également beaucoup dans ce domaine. L'objectif d'augmenter les connaissances scientifiques du public n'est pas la grande ou la vraie raison. Non ça ne l'est pas. Pour un géoparc, réussir l'évaluation vient avant tout. (Traduction libre d'un extrait d'entretien)

Dans le même esprit, Du et Girault (2019, p. 258) rappellent que pour qu'un territoire obtienne en Chine le label "Géoparc national" (label indispensable pour pouvoir espérer par la suite obtenir le label UGG), il doit absolument posséder un musée qui va devenir le point d'orgue de la médiation du territoire. Cependant, malgré la gratuité d'entrée, ces auteurs soulignent qu'il y a le plus souvent très peu de visiteurs dans les musées de géoparcs. Ainsi il n'y aurait eu que 60000 visiteurs au musée du Géoparc mondial de Dali Mt Cangshan sur 980000 visiteurs de ce géoparc et seulement 1600 entrées au musée du Géoparc mondial de Mt Kunlunshan sur 3,45 millions de visiteurs. D'autres auteurs soulignent cet antagonisme entre la nécessité de répondre aux objectifs de l'évaluation et celle de prendre en compte les aspirations des visiteurs. Xu 
et coll. (2013) précisent ainsi que les musées de géoparcs d'une part et d'autre part les panneaux d'interprétation scientifique mis en place afin d'obtenir le label UGG, sont rarement utilisés. Plusieurs responsables nous ont même affirmé que certains musées de géoparcs n'étaient ouverts que pour les évaluateurs internationaux ou nationaux ce qui est corroboré par Anze Chen qui affirme qu'un "grand nombre des musées de géoparcs en Chine ne sont ouverts que pour obtenir le label ; une fois labellisé, le musée est fermé sans autorisation » (Anze Chen, 2012, p. 63).

\section{En guise de conclusion}

Nous avons montré que le développement des activités d'interprétation du patrimoine naturel en Chine est le fruit d'une hybridation culturelle. Ces activités répondent en effet à des injonctions politiques nationales clairement affirmées pour lutter contre les croyances anciennes. Cette impulsion d'une politique de "science popularization », qui est promue par les intellectuels chinois, ne peut cependant pas s'appuyer de nos jours sur des professionnels suffisamment formés aux techniques de communication scientifique. Cela engendre donc des problèmes de réception au niveau des touristes locaux qui, in fine, se désintéressent des structures muséales d'autant plus qu'ils privilégient majoritairement une approche immersive et sensible de la nature. Au niveau international, les injonctions de l'UNESCO induisent des effets pervers puisque les travaux d'amélioration des présentations, qui sont réalisés selon le calendrier quadriennal d'évaluation, sont basés principalement sur la grille d'évaluation exogène retenue à cet effet. Les attentes des touristes chinois sont malgré tout prises en compte par l'aménagement de points d'accès faciles et la valorisation, au sein des géoparcs, de nombreux anthroposites comme les roches gravées et les pavillons anciens.

Nous pouvons cependant nous demander dans quelle mesure l'instance des UGG, en respect de la diversité culturelle, ne pourrait-elle pas privilégier l'évaluation des projets de développement des géoparcs en termes de production de connaissances hybrides (Fraser et Lepofsky, 2004, cités dans High et Nemes, 2007), soit en termes de compréhension partagée à travers des interactions mise en place par le projet.

\section{BIBLIOGRAPHIE}

Ballantyne, R., Hughes, K., Ding, P. et Liu, D. (2014). Chinese and international visitor perceptions of interpretation at Beijing built heritage sites. Journal of Sustainable Tourism. 22(5), 705-727.

Blamey, R. K. (2001). Principles of Ecotourism. Dans D. B. Weaver (dir.). Encyclopaedia of Ecotourism (p.5-22). Wallingford, UK : CABI.

Buckley, R. et al. (2008) Shengtai Lüyou : Cross-Cultural Comparison in Ecotourism, Annals of Tourism Research, 35(4), 945-968.

Choe, Y., M. A. Schuett, et K. W. Sim. (2017). An analysis of first-time and repeat visitors to Korean national parks from 2007 and 2013. Journal of Mountain Science 14(12), 2527-2539. 
Chen, A. (2012). Several discussions on museum of geopark [地质公园博物馆建设若干问题探讨]. Dans l'acte du collogue Annual conference of committee geotourism and geopark-China geological society \& seminar on construction and touristic development of Zhangye Danxia National Geoaprk [中国地 质学会旅游地学与地质公园研究分会年会暨张掖丹霞国家地质公园建设与旅游发展研讨会] (p. 61-64), Août 2012, Zhangye.

Chen, A., Lu, Y. et Ng, Y. C. Y. (2015). The Principles of Geotourism. Berlin Heidelberg : Springer. Donohoe, H.M., et Needham, R.D. (2006). Ecotourism : The evolving contemporary definition. Journal of Ecotourism, 5(3), 192-210.

Dowling, R. K. et Newsome, D. (2006). Geotourism : sustainability, impacts and management. Elsevier.

Du Y., et Girault Y. (2019). La médiation architecturale des musées de géoparcs en Chine : entre tensions et hybridation des cultures. In Girault Y. (dir.).Les géoparcs mondiaux UNESCO : une mise en tension entre développement des territoires et mise en valeur du patrimoine (p. 253277). Londres : ISTE Éditions.

Du Y. et Girault Y. (2018). A Genealogy of UNESCO Global Geopark : Emergence and Evolution. International Journal of Geoheritage and Parks, 2018, 6(2), pp 1-17.

Elliott, M. C. (2006). La Chine moderne : Les Mandchous et la définition de la nation, Annales. Histoire, Sciences Sociales, (6), 1447-1477.

Elvin, M. (2004). The Retreat of the Elephants : An Environmental History of China. New Haven et London : Yale University Press.

Frey, M.-L. et al. (2006).Geoparks a regional, European and global policy. Dans Dowling, R. K. et Newsome, D. (dir.). Geotourism (p.95-117). Elsevier.

Geng, Y. et Wang, J. (2013). Research on Earth Science Popularization of Geopark Museum : Status and Its Enlightenment [地质公园博物馆地学科普的现状及后示], Journal of Anhui Agrii. Sci. [安徽 农业科学], (14), 6365-6367。

Gonzalez-Tejada, C., Du Y., Read M., Girault Y. (2017). From nature conservation to geotourism development : Examining ambivalent attitudes towards UNESCO directives with the global geopark network. International Journal of Geoheritage, 5(2), 1-20.

Hou L.S. et Xu X.G. (2004). Meaning and Values of National Geopark [国家地质公园的内涵及其价 值特征]. Management of geological technologies [地质技术经济管理], 26(1), 48-50.

Hwang, S.-N., C. Lee, and H.-J. Chen. (2005). The Relationship among Tourists' Involvement, Place Attachment and Interpretation Satisfaction in Taiwan's National Parks. Tourism Management, 26 (1), 143-56

Landsberger, S. (2005). Socialist spiritual civilization. Dans Davis (dir.). Encyclopedia of Contemporary Chinese Culture. Routledge.

Li, Y. and Yang, G. (2012). An Analysis of Current Situation of Science Popularisation in Chinese Geoparks. [浅析我国地质公园的科普现状]. Dans Proceeding of the $19^{\text {th }}$ national conference on science communication \& 2012 international forum on S\&T communication in Asia-Pacific [科学传播创新与科学 文化发展一一中国科普理论与实践探索一一第十九届全国科普理论研讨会暨2012亚太地区科技传 播国际论坛论文集] (p. 161-168). Août 2014, Beijing.

Lin, S. (2009). The History of “Ziran” and “Nature” in Translation [新「自然」考]. Bulletin of the Department of Chinese Literature N.T.U [臺大中文學報, 31, 269-310.

Pieterse, J. N. (2009). Globalization and Culture: Global Mélange. 2nd Edition. Maryland : Rowman \& Littlefield Publishers, Inc. 
Prosser, C. D., Bridgland, D. R, Brown, E. J, Larwood, J. G. (2011). Geoconservation for science and society : Challenges and opportunities. Proceedings of the Geologists' Association, 12, 337-342.

Romeril, M. (1985). Tourism and the environment towards a symbiotic relationship : Introductory Paper. International Journal of Environmental Studies, 25(4), 215-218.

Shi, S. and Zhang, H. (2012). Policy Perspective on Science Popularisation in China. Dans Schiele, B., Claessens, M., et Shunke, S. (dir.). Science Communication in the World: Practices, 65 Theories and Trends (p.81-94). Springer.

Shue, V. (2004). Legitimacy crisis in China? Dans Gries et Rosen (dir.). State and Society in 21stcentury China : Crisis, Contention, and Legitimation (p. 24-49). New York : RoutledgeCurzon.

Sofield, T.H.B., et Li, F.M.S. (2007). China : Ecotourism and cultural tourism : Harmony or dissonance ? Dans J. Higham (dir.), Critical issues in ecotourism : Confronting the challenges (p. 368385). London : Elsevier Science \& Butterworth Heinemann.

Songster, E. E. (2004). A Natural Place for Nationalism : The Wanglang Nature Reserve and the Emergence of the Giant Panda as a National Icon. These de doctorat. University of California, San Diego.

Stokes, A. M., Cook, S. D., Drew, D. (2003). Geotourism : The New Trend in Travel. Whashington D.C. : Travel Industry Association of America et National Geographic Traveler Magazine.

Weaver, D.B., et Lawton, L.J. (2007). Twenty years on : The state of contemporary ecotourism research. Tourism Management, 28(5), 1168-1179.

De Wever P., Cornée A., Egoroff G., Collin G., Duranthon F., Lalanne A., De Kermadec C., Lucet S. (2019). Patrimoine géologique : notion, état des lieux, valorisation. NATURAE, 2019 (1), 1-58

Weller, R. P. (2006). Discovering Nature : Globalization and Environmental Culture in China and Taiwan [Kindle edi]. Cambridge University Press.

Wu, B., and L. Cai. (2006). Spatial Modelling : Suburban Leisure in Shanghai. Annals of Tourism Research, 33 (1), 179-98.

Wu, G. (2016). Science communication in contemporary China' [当代中国的科学传播], Journal of Dialectics of Nature [自然辩证法通讯] , 38(2), 1-6.

$\mathrm{Xu}, \mathrm{H}$. et al. (2013). Effective environmental interpretation at Chinese natural attractions : the need for an aesthetic approach. In Journal of Sustainable Tourism. Taylor \& Francis Group, 21(1), 117-133.

Xu, H. et al. (2014). Attaining harmony : Understanding the relationship between ecotourism and protected areas in China. Journal of Sustainable Tourism, 22(8), 1131-1150.

\section{NOTES DE FIN}

1. Ce travail a été réalisé dans le cadre du projet GEOPARK financé par le programme de recherche et d'innovation Horizon 2020 de l'Union européenne sous le numéro de la convention de subvention no. 644015 .

2. Cette analyse est issue du travail de recherche doctorale en cours de Yi Du réalisé sous la direction d'Yves Girault

3. Les « Lettrés » désignent des fonctionnaires érudits dans la Chine impériale.

4. Gravures calligraphiques sur les falaises rocheuses ou sur des blocs de rochers.

5. Les montagnes sont vénérées en Chine par les taoïstes, comme des divinités et par les bouddhistes, comme des résidences des divinités. 
6. Nom donné, à partir de 1920 en URSS, à un courant en agronomie et en génétique défendus par Lyssenko et basé sur des présupposés idéologiques.

7. Les vieilles idées, les vieilles cultures, les vieilles coutumes et les vieilles habitudes. La destruction des Quatre Vieux pendant la Révolution Culturelle de 1966-1976 pourrait être interprétée comme l'apogée d'un long processus de luttes pour des valeurs euro-centriques contre la tradition chinoise (principalement confucianiste), depuis le mouvement de la Nouvelle Culture qui a débuté en 1915.

8. NPS https://www.nps.gov/orgs/1207/03-05-2019-visitation-numbers.htm [consulté le 26 mars 2019])

9. Document UNESCO 156 EX / 11 Rev. « Programme géoparcs de l'UNESCO - nouvelle initiative pour promouvoir un réseau mondial de géoparcs permettant de préserver et de mettre en valeur des zones présentant des caractéristiques géologiques particulières ». Ce document est établi à la suite de la réunion d'information organisée le 23 mars 1999 par le Secteur des sciences exactes et naturelles à l'intention des délégués permanents et des observateurs auprès de l'UNESCO.

10. 12 réserves nationales, 33 réserves régionales, 9 réserves départementales et 32 réserves cantonales.

11. Le Plan étant un document interne non accessible, nous avons trouvé les chiffres cités dans Liu Hao (2007). consultable en ligne http://cn.globalgeopark.org/thesis/4629.htm

12. Connaître la structure de la Terre, planète vivante en constante évolution depuis 4,6 milliards d'années, comprendre son fonctionnement, son histoire et son évolution sont les objectifs de ce que l'on appelle les Géosciences.

13. Le terme « civilisation spirituelle » ou exactement « civilisation spirituelle socialiste » est un terme politique. «Conformément aux changements sociaux de la réforme, le Parti-État a tenté de formuler de nouvelles valeurs et de nouveaux concepts visant à sensibiliser les Chinois à la pensée idéologique. L'élément le plus important de ce processus d'apprentissage est la construction d'un système de civilisation spirituelle socialiste » (Landsberger, 2005, p. 769)

14. Sauf pour l'item «Geotourisme » (code D), dont le total des points des 14 questions/ est 970. Le document indique cependant toujours un total de 1000 points disponibles pour cet item. Il s'agit sans doute d'une erreur de la part de UNESCO.

15. version 11 février 2016, téléchargeable sur le site officiel de l'UNESCO http:// www.unesco.org/new/fileadmin/MULTIMEDIA/HQ/SC/pdf/EN_UGGEvaluation_DocA_Selfevaluation_FINAL_12Feb2016_PR.xls [consulté le 25 avril 2019]

16. version 9 janvier 2018, téléchargeable sur le site officiel de l'UNESCO http://www.unesco.org/ new/fileadmin/MULTIMEDIA/HQ/SC/pdf/UGG_Evaluation_DocB_Progress-eval_2018_EN.xls [consulté le 25 avril 2019]

17. Le Programme international de géosciences et des géoparcs (PIGG) est mis en œuvre à travers deux activités : le Programme international de géosciences (PICG), partenariat de coopération avec l'Union internationale des sciences géologiques (UISG), et les géoparcs mondiaux UNESCO.

18. Entretiens réalisés par Yi Du dans le cadre de sa recherche doctorale.

19. Le label UGG est accordé à un territoire pendant quatre années après lesquelles un processus de revalidation a lieu. Selon le rapport d'évaluation, si le territoire correspond toujours aux critères de de l'UGG, il reçoit une «carte verte " et garde le label UGG pendant une nouvelle période de quatre ans. Quand les critères de l'UGG ne sont plus respectés, une « carte jaune » est donnée au territoire et ce dernier aura 2 ans pour prendre en compte les recommandations. Au bout de deux ans si le territoire n'arrive toujours pas à atteindre les critères retenus, l'UNESCO donnera finalement la « carte rouge » pour retirer le label UGG au territoire. 
20. Citation dans l'article de presse « Dette de communication scientifique ! Trois grand géoparcs mondiaux avertis par la carte jaune». http://scitech.people.com.cn/n/2013/0130/ c1007-20370149.html consulté le 27 fév 2018.

21. Expression employée par Chen Anze, l'un des fondateurs du géoparc chinois et Président de l'association du géotourisme chinois. Propos tiré de l'article « Géoparc, comment pérenniser ses charmes?» [dizhi gongyuan, zenyang cai neng meili yong cun?], publié le 25 août 2005 dans China Land and Ressources News. http://gtszb.gtzyb.com/gtszb/resfile/2005-08-25/04/04.pdf (consulté le 27 fév 2018).

22. Connaître la structure de la Terre, planète vivante en constante évolution depuis 4,6 milliards d'années, comprendre son fonctionnement, son histoire et son évolution sont les objectifs de ce que l'on appelle les Géosciences.

23. Le géopatrimoine (équivalent de geoheritage en anglais) relève de faits géologiques qui ont des importances globales (mondiales, nationales ou locales) et de sites géologiques qui représentent des phénomènes (volcanisme, ségrégation magmatique, métamorphisme, altération, sédimentation ...) et/ou qui témoignent de l'histoire de la Terre (paléontologie, tectonique globale, climat, niveau marin ...). Il concerne des objets de toute taille (du paysage à la taille du minéral) qui sont intrinsèquement (par leur valeur propre) ou extrinsèquement importants, par le regard que l'on porte sur eux, c'est-à-dire culturellement. De Wever P., Cornée A. et al. ( 2019).

\section{RÉSUMÉS}

$\mathrm{Au}$ cours de trois périodes historiques majeures en Chine, l'évolution des rapports à l'environnement a été largement induite par des pratiques culturelles spécifiques à l'Asie (en particulier le confucianisme et le taoïsme) et par l'intégration progressive d'éléments de la culture occidentale. Dans le cadre de la revalorisation du tourisme, les parcs naturels ont développé des activités écotouristiques. Cette ouverture s'est accompagnée en Chine, de nouveaux défis et de nouvelles opportunités car si les touristes chinois ont un intérêt très fort pour les zones naturelles et sont toujours influencés par leur culture traditionnelle, des autorités privilégient le développement de la «science popularization ». Enfin, depuis les années 2000, la Chine a labellisé de très nombreux géoparcs nationaux et a rejoint progressivement le réseau mondial des géoparcs qui impose également ses propres objectifs. Après avoir présenté ce contexte très spécifique, nous proposons de souligner les tendances actuelles des activités d'interprétation de la nature au sein de ces géoparcs.

Over three major historical periods in China, the evolution of relationships with the environment was largely induced by cultural practices specific to local context (Confucianism, Taoism...) and by the integration of other cultural practices. As in the context of the revival of the tourism, nature-based parks have developed tourist activities, some of which are based on ecotourism. This opening of the ecotourism market in China has brought new challenges and opportunities for park managers because if Chinese tourists have a strong interest in natural areas and are still influenced by the traditional literati culture, authorities favor the development of science popularization. Finally, since the 2000s, China has labelled a large number of National Geoparks and has gradually joined the global network of geoparks, in which also imposes its own objectives. After presenting this very specific context, we propose to highlight current trends in nature interpretation activities within these geoparks. 
INDEX

Mots-clés : interprétation, géoparc, géotourisme, écotourisme, UNESCO

Index géographique : Chine

Keywords : interpretation, geopark, geotourism, ecotourisme, science popularization, UNESCO

\section{AUTEURS}

YI DU

Yi Du est doctorante au Muséum national d'Histoire naturelle au sein de l'UMR 208 Patrimoines locaux, environnement et globalisation (MNHN/IRD). Dans sa recherche doctorale elle analyse les modalités d'appropriation du label « Géoparc mondial UNESCO » en Chine sous le prisme de l'hybridation des cultures.

\section{YVES GIRAULT}

Yves Girault est professeur au Muséum national d'Histoire naturelle, coordinateur général du projet européen GEOPARK (H2020-MSCA-RISE-644015), co-éditeur en chef de la revue "International Journal of Geoheritage ands Parks" Beijing Normal University (Chine) et membre du Centre de recherche en éducation et formation relatives à l'environnement et à l'écocitoyenneté à l'UQAM (Montréal). Ses travaux portent notamment sur l'étude de l'évolution des discours scientifiques concernant les rapports Hommes - natures - sociétés dans le champ particulier du musée (analyse des enjeux socio-politiques, des représentations identitaires des acteurs, du mode d'énonciation et des modes de représentations muséographiques). 